\title{
La variación textual y su visualización en las ediciones digitales de textos medievales Textual variation and its visualization in digital editions of medieval texts
}

Resumen: Una de las características principales de la transmisión textual de obras medievales en lenguas vernáculas es la elevada variabilidad de los textos conservados. Si bien se trata de un fenómeno conocido desde siempre, desde la discusión en torno a la new philology en los ' 90 el editor de textos medievales está obligado a reflejarlo en su labor. La dificultad reside en jerarquizar las variantes con criterios objetivos y en presentar las más relevantes al lector de manera inteligible. La dificultad es aún mayor cuando se trata de variaciones en el número y en el orden de versos. Las ediciones electrónicas tienen altísimas capacidades para reflejar la complejidad de la transmisión textual, pero precisan para eso de una interfaz que sepa leer y mostrarla. Nuestra presentación expondrá el problema y revisará diferentes herramientas que han propuesto soluciones. Finalmente, sacaremos algunas conclusiones acerca del alcance y las limitaciones de las herramientas actualmente disponibles.

Palabras clave: edición digital; variación textual; new philology; visualización; colación automática.

Abstract: A main feature of the textual transmission of medieval vernacular works is the high degree of variation in the extant witnesses. Although this issue has been known since the rise of new philology in the 90s, the editor of medieval texts is forced to reflect it in his or her work. The difficulty lies in ranking the variants with objective criteria and presenting the reader with the most relevant of these in an intelligible manner. The difficulty is even greater when variations occur in the number and order of the verses.

Electronic editions have a great capacity to reflect the complexity of textual transmission, but they require an interface that can read and illustrate this complexity for the reader. This paper outlines the problem here and reviews a number of tools that offer potential solutions; some conclusions are drawn regarding the limitations and hence the usefulness of currently available tools.

Gustavo Fernández Riva, Universidad de Buenos Aires/CONICET

Victor Millet, Universidade de Santiago de Compostela

๖ Open Access. () 2019 Fernández Riva and Millet, published by De Gruyter. (c) BY-NC-ND This work is licensed under the Creative Commons Attribution-NonCommercial-NoDerivatives 4.0 License. https://doi.org/10.1515/9783110585421-006 
Keywords: Digital Editions; Textual Variation; New Philology; Visualization; Automatic Collation.

\section{Problemática filológica}

Una de las características principales de la transmisión textual de obras medievales en lenguas vernáculas es la elevada variabilidad de los textos conservados. En Alemania -en cuya tradición literaria manuscrita nos centramos aquí por ser la que mejor conocemos, si bien nuestras observaciones son fácilmente trasvasables, con los debidos ajustes, a las literaturas románicas- la novela caballeresca y de materia clásica cuentan con manuscritos con grados diversos de variación, unas veces elevados y relevantes, otras veces menores; llama la atención en este contexto que casi todas las principales obras (p.ej. el Parzival o el Tristán) cuentan con "versiones abreviadas", es decir versiones que son mil o dos mil versos más breves y si bien ello no siempre afecta al contenido esencial del relato no cabe duda de que se pierden matices. En el contexto de la poesía heroica cabe resaltar que el Cantar de los Nibelungos se escribe, al parecer desde el propio entorno del autor anónimo, en dos versiones de distinto enfoque, sin que podamos decir cuál es la genuina, es decir que la variación parece partir del propio origen del texto. En general, la poesía épica parece haber preferido la reedición del material que la copia, ya que es raro encontrar un manuscrito que no introduzca importantes cambios en el relato, de modo que hablamos aquí de otro tipo de variabilidad. Algo parecido ocurre en la narrativa breve, si bien es cierto que, al igual que en los demás géneros, unos textos parecen, desde un principio, más expuestos a la variación intensa que otros, aunque no sabemos exactamente por qué. En la lírica alemana, la variación podría considerarse relativamente normal en el plano del contenido, si bien la variación en el número y orden de las estrofas es más elevada en los cancioneros alemanes que en los románicos.

En todo caso, la variación es un fenómeno conocido y reconocido desde los inicios de la edición filológica. Si en 1815 los hermanos Grimm anotaron a modo de notas a pie de página las principales variantes de un texto de Hartmann von Aue que editaron y que conocían sólo de dos manuscritos, doce años más tarde su amigo Karl Lachmann, formado en las filologías clásica y bíblica, introdujo el modelo del aparato crítico. ${ }^{1}$ El aparato crítico tiene la virtud de poder concentrar, en un espacio reducido y secundario de la página, información acerca de variantes de muchos

1 En las ediciones de Walther von der Vogelweide (Lachmann 1827) y de Hartmann von Aue (Benecke/Lachmann 1827). Si bien existen ejemplos de anotación de la variación similares al 
manuscritos. Sin lugar a dudas, cuando hay que editar en formato de libro un texto conservado en cincuenta manuscritos resulta imprescindible concentrar las variantes en un aparato lo más denso posible, a fin de que el texto editado no quede sepultado bajo la multitud de textos conservados. Sin embargo, el precio que paga el aparato crítico por esa gran capacidad de condensación es elevado: puesto que uniformiza las variantes y reduce el espacio, dificultando a veces la lectura distinta del verso o frase completos, hace que las variantes importantes se vean menos. A veces, variantes realmente relevantes, donde el texto de determinado manuscrito o incluso de un grupo de manuscritos dice no solo algo distinto, sino algo significativamente distinto, es decir que tiene consecuencias para la comprensión e interpretación del texto, quedan escondidas en el revoltijo de cifras, siglas y palabras sueltas del aparato crítico. El texto editado luce, pero la información de los demás textos, aunque presente, queda fragmentada y no es comprensible en su conjunto.

Mientras la filología creía saber o poder deducir cuál era el texto más próximo al autor, o mejor aún, cuál había sido la verdadera intención del autor, el problema tenía una relevancia menor. La alternativa consistía en la edición según el manuscrito guía, que institucionalizó Gustav Roethe en $1904 .{ }^{2}$ Pero en el fondo el problema era el mismo: o bien la información sobre los demás manuscritos se obviaba, o bien había que concentrarla en poco espacio y se perdía la visibilidad. De una u otra manera, dado que se entendía que el manuscrito editado era el mejor de los conservados, las variantes tenían una importancia menor porque se consideraban como desviaciones, como errores, como texto "no auténtico".

En los años "60 se inició en la filología alemana una discusión de vasto alcance sobre la edición de textos medievales. El texto editado, en palabras de Karl Stackmann (1964), dejaba de ser una autoridad para convertirse en una tarea, es decir que ya no se le veía como un monolito sino como el punto de partida de un proceso de trabajo. Stackmann demostró que las condiciones que exigía el método lachmanniano (un único texto de autor, trasmisión vertical, claridad de los “errores”, etc.) casi nunca se daban en textos medievales. Ello por un lado relativizaba la autoridad del editor, que ahora resultaba falible, y por el otro revalorizaba las variantes como elemento con el que la crítica textual podía y debía seguir trabajando. Y eso exigía resaltar aquellas variantes que realmente eran significativas frente a aquellas que no lo eran. Aparte del problema teórico estaba la cuestión pragmática de las ediciones en soporte papel. En la década de

aparato crítico en antiguas ediciones bíblicas, es recién a principios del siglo XIX que se establece y vuelve convencional la forma del aparato crítico moderno.

2 En la colección Deutsche Texte des Mittelalters. Aunque también Karl Lachmann trabajaba con un manuscrito guía, quienes continuaron su escuela tendieron hacia una cada vez mayor "reconstrucción" de un supuesto original. 
los '80 se ensayaron diversas opciones, tal como el resaltar las variantes importantes dentro del aparato crítico dedicándoles líneas propias o bien crear dos aparatos críticos, uno para las variantes relevantes y otro para las demás. Ambas seguían generando insatisfacción, dado que la variante relevante a menudo hay que leerla en el contexto de toda una frase, que puede abarcar varios versos o líneas.

Esta situación hizo que en Alemania se reaccionara de manera algo más reservada ante la postura de la llamada new philology presentada por Bernard Cerquiglini (1989), porque si bien el texto medieval es variación, como decía Cerquiglini, en Alemania estaba claro que la variación textual que reflejan los manuscritos mayoritariamente no es reescritura, es decir generadora de nuevo significado. Mucha mejor aceptación gozó el planteamiento de Stephen Nichols (1997) que situaba el manuscrito en el centro de la cuestión como cruce de líneas discursivas que proceden bien del autor, bien de nuevas lecturas. Cada manuscrito es entonces el testimonio de una lectura determinada del texto, cada variante es una potencialidad del texto y aunque ya no nos atrevemos a afirmar cuál de ellas pudo haber sido la que ideó el autor de la obra, ello ya no importa porque hemos dejado de buscar el texto del autor, inalcanzable, y a cambio hemos descubierto que cada uno de los textos alberga o puede albergar matices, diferenciaciones o posibilidades que en algún momento lectores o copistas históricos vieron en ese texto.

Pero aunque la variante textual haya dejado de ser molestia para convertirse en riqueza y valor a nivel teórico, todavía no se ha solucionado del todo el problema de su visualización a nivel pragmático. Cerquiglini abogaba por el potencial de las ediciones electrónicas. No cabe duda de que cualquier edición electrónica tiene el potencial de albergar toda la transmisión textual de una obra determinada sin ocupar más espacio físico, lo que significa que en teoría puede mostrar al mismo tiempo todos los manuscritos y todas sus variantes. Pero la realidad es que, por un lado, tanto las pantallas como sobre todo la capacidad visual del ojo humano tienen una limitación y, por el otro, tanto la docencia como la investigación demandan, a nivel pragmático y en la mayoría de ocasiones, un texto, no un bosque de textos, es decir, comprensibilidad, inteligibilidad y citabilidad.

Nuestras reflexiones sobre la variación textual y la capacidad de las ediciones electrónicas para representarla y analizarla surgen, en buena medida, de la necesidad de encontrar una forma adecuada para una edición concreta, la de Der arme Heinrich de Hartmann von Aue (Der Arme Heinrich digital). Entre las diferentes versiones que se conservan de ese texto (dos principales, representadas por sendos manuscritos completos, y trazos de intensa variación también en los cinco fragmentos conservados), existen numerosas variantes, especialmente 
trasposiciones y versos que están presentes solo en una de ellas. En ese contexto, surge como reto encontrar la manera más adecuada de representar este estado textual. Lo mismo ocurre en el siguiente proyecto considerado, la edición de los relatos breves de Konrad von Würzburg, cuyas soluciones abordaremos más abajo.

\section{Formas digitales de análisis de la variación textual}

Desde los orígenes de las Humanidades Digitales han existido diversos intentos de abordar el problema de la variación textual. ${ }^{3}$ Una de las primeras herramientas que se desarrollaron fue Collate y su sucesor CollateX a partir de 2010. CollateX utiliza un algoritmo que alinea las palabras de los diferentes testimonios y ofrece diferentes resultados posibles. Para el trabajo de colación básica ofrece un output en forma de tabla (Figura 5.1):

\begin{tabular}{|c|c|c|c|c|c|c|c|}
\hline W1 & E1 & gato & blanco & duerme & sobre & los & libros \\
\hline W2 & E1 & perro & & duerme & sobre & el & sofá \\
\hline
\end{tabular}

Figura 5.1: Ejemplo de CollateX.

Este output se acerca mucho a la forma que podían adoptar las colaciones en el mundo de la página impresa (o manuscrita). La gran ventaja es el algoritmo que alinea automáticamente los términos y los colores que ayudan a reconocer concordancias y variantes. Sin embargo, esta visualización no intenta explotar realmente las posibilidades de la computación; se encuentra todavía fuertemente ligada al mundo analógico.

Una de las visualizaciones más originales surgidas de la tecnología informática que intenta aprovechar las ventajas de la visualización en pantalla son los variant graphs. El grafo es una estructura de la información particular, que fue propuesta por Schmidt/Colomb (2009) como una manera de superar

3 Para un estado de la cuestión pueden consultarse la monografía de Pierazzo (2015) y la colección de artículos de Driscoll/Pierazzo (2016). En este artículo nos centraremos en el problema de la visualización de la variación para un tipo de lectura humana sobre la pantalla y dejaremos de lado el complejo problema de la medición del nivel de variación. 
los problemas de la codificación de la variación textual en TEI. Desde nuestra perspectiva, no importa tanto el grafo como estructura para codificar y almacenar la variación textual, sino como forma de visualizarla. CollateX ofrece la posibilidad de generar variant graphs y visualizarlos en pantalla que es también utilizada por StemmaWeb (Figura 5.2):

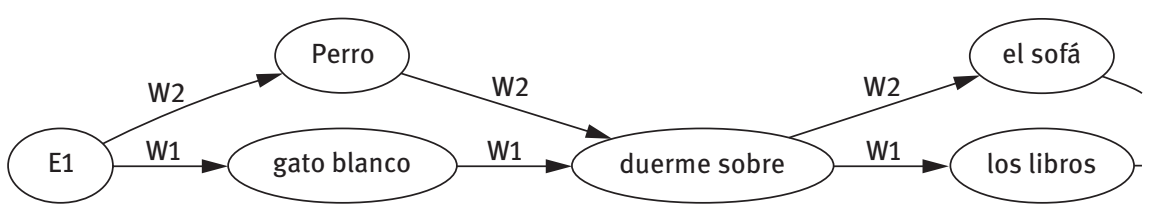

Figura 5.2: Ejemplo de grafo de variantes.

Este tipo de grafo es posible solo en un ámbito digital, no porque no se pueda dibujar, sino porque hacerlo en formato impreso demandaría un esfuerzo no justificable, mientras que la automatización digital los crea con facilidad. La ventaja de éste sobre la tabla es que ofrece mucha información acerca del nivel de variación presente en el fragmento en su propia forma. Es decir, todas las tablas se ven más o menos iguales, más allá de la variación presente en el texto. En cambio, un grafo con más variación presenta más líneas bifurcantes que uno con menos. Los usuarios pueden hacerse una idea del nivel de variación con solo echar un vistazo al grafo. Esta visualización también permite indicar trasposiciones, un fenómeno siempre muy difícil de representar (Figura 5.3):

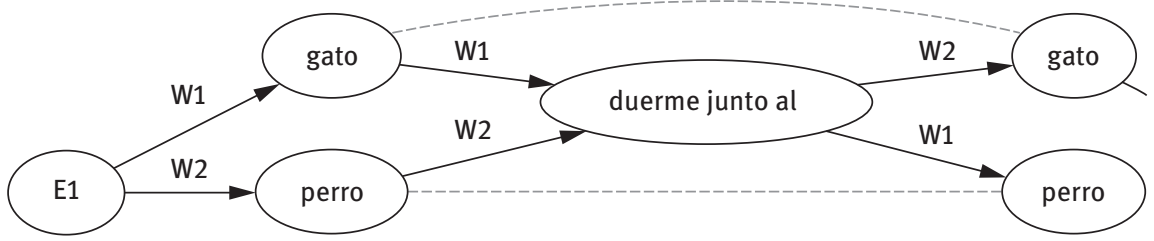

Figura 5.3: Ejemplo de grafo con transposición.

El problema del variant graph consiste en que marcar la transposición puede generar confusión si no hay una lectura muy cuidadosa. Como se ve en la Figura 5.3, para marcar el fenómeno los dos testimonios cambian de lugar relativo (W1 pasa a estar debajo de W2) y esto puede causar equivocaciones si no se lee atentamente.

El proyecto Traviz desarrolla grafos similares a estos, pero que aprovechan al máximo la posibilidad de la presentación en colores (Figura 5.4): 


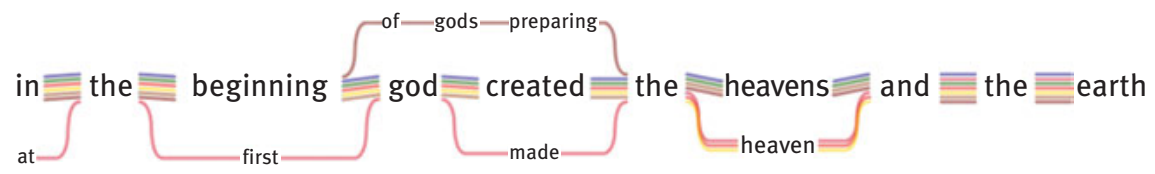

Figura 5.4: Ejemplo de Traviz.

Traviz posee la ventaja de ser interactivo, es decir, que el usuario puede realizar acciones que alteran la imagen y permiten focalizar sobre ciertos testimonios al posar el cursor sobre una palabra. Como se ve en el ejemplo siguiente, al posar el cursor sobre la palabra made, presente en un solo testimonio, se marca solo la línea correspondiente a ese testimonio (Figura 5.5):

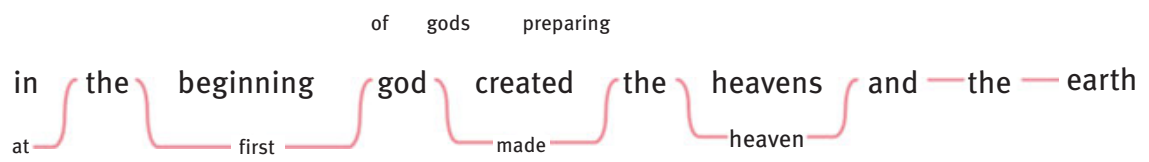

Figura 5.5: Ejemplo de Traviz con un solo testimonio seleccionado.

Este tipo de visualizaciones son útiles a nivel de un verso o una oración particular, pero resultan poco fructíferas para analizar textos más largos. Esto se debe a que su complejidad gráfica puede ser interpretada fácilmente para un fragmento específico pero puede sobrecargar la capacidad de lectura en un texto largo.

Una forma de visualizar mejor la variación a un nivel superior del verso o la oración es Juxta Commons. Esta tecnología toma diferentes testimonios, los colaciona (utilizando algoritmos similares a CollateX) y ofrece diferentes visualizaciones. Es posible, también, exportar el resultado de la colación en formato TEI, aunque el resultado no es muy confiable en casos complejos. La forma de visualización más básica es el heatmap, que resalta el texto que presenta variantes en otros testimonios. Al hacer click sobre el texto resaltado se abre una pequeña ventana con la variante en cuestión (Figura 5.6):

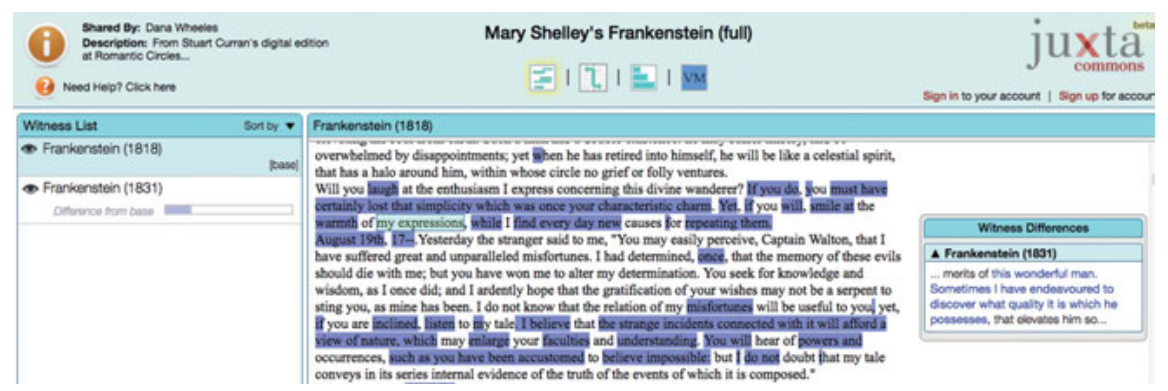

Figura 5.6: Ejemplo 1 de Juxta Commons. 
Juxta Commons también ofrece la posibilidad de ver los dos textos de manera sinóptica, resaltando y estableciendo conexiones entre los lugares donde hay variación (Figura 5.7):
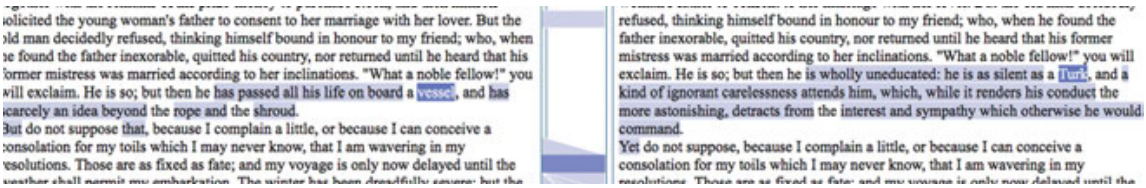

Figura 5.7: Ejemplo 2 de Juxta Commons.

Una herramienta que ofrece una visualización muy similar a esta es MEDITE, ${ }^{4}$ desarrollada por el Institut des Textes et Manuscrits Modernes del CNRS y el Laboratoire d'Informatique de Paris VI, pensada originalmente para análisis de crítica genética. La visualización de este programa posee diferentes colores para distinguir claramente entre transformaciones, por un lado, y añadidos o supresiones, por otro. Además, permite muy fácilmente alterar parámetros (como diferenciar mayúsculas o considerar signos diacríticos) y generar una lista de todas las variaciones identificadas (Figura 5.8): ${ }^{5}$

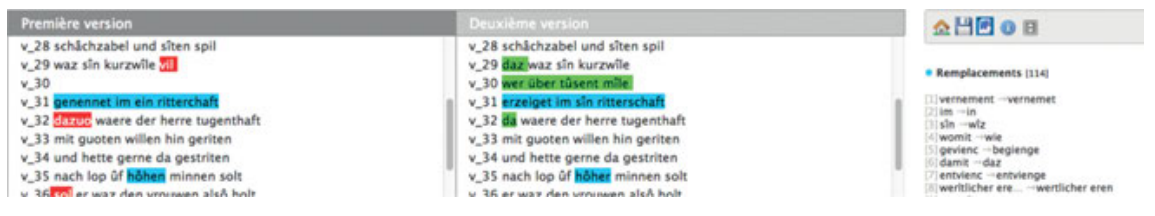

Figura 5.8: Ejemplo de MEDITE.

Finalmente, vale la pena mencionar otra visualización ofrecida por Juxta: el "histogram”, un gráfico que representa el nivel de variación en diferentes fragmentos del texto (Figura 5.9):

4 http://obvil.lip6.fr/medite/about_medite.html.

5 Una característica sobresaliente de esta herramienta es que además permite descargar los resultados del análisis como una página web completa o en un archivo de texto con tablas que explicitan distintos tipos de información (listas de fragmentos insertados, elididos, reemplazados, compartidos). 


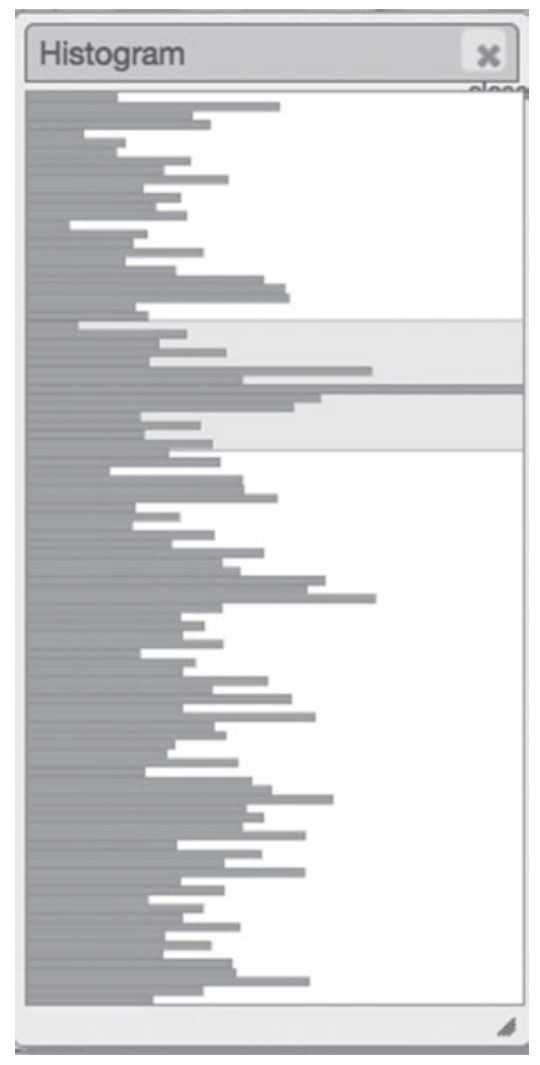

Figura 5.9: Ejemplo de histogram.

Juxta Commons es especialmente productivo para analizar textos en prosa y con un bajo grado de variación. Por ejemplo, es ideal para comparar diferentes ediciones de libros impresos, lo que se aprecia en los ejemplos elegidos pertenecientes a la colación de las ediciones de 1818 y 1831 de Frankenstein de Mary Shelley. En estos casos es posible leer claramente un texto y comparar sencillamente los cambios, agregados o sustracciones. Para casos más complejos, como los textos medievales, la visualización de Juxta Commons puede resultar confusa y, además, no es adecuada para representar fenómenos característicos de la poesía medieval como la trasposición o el agregado de versos.

Una visualización que intenta representar la variación al nivel estructural para textos medievales es el stream graph, desarrollado por Jänicke/Wrisley (2017). En esta visualización sinóptica, los testimonios se ubican verticalmente y curvas de resaltado alinean los versos correspondientes. Al ubicar el cursor sobre uno de ellos, la línea se resalta de un color más fuerte, lo que facilita la lectura (Figura 5.10). 


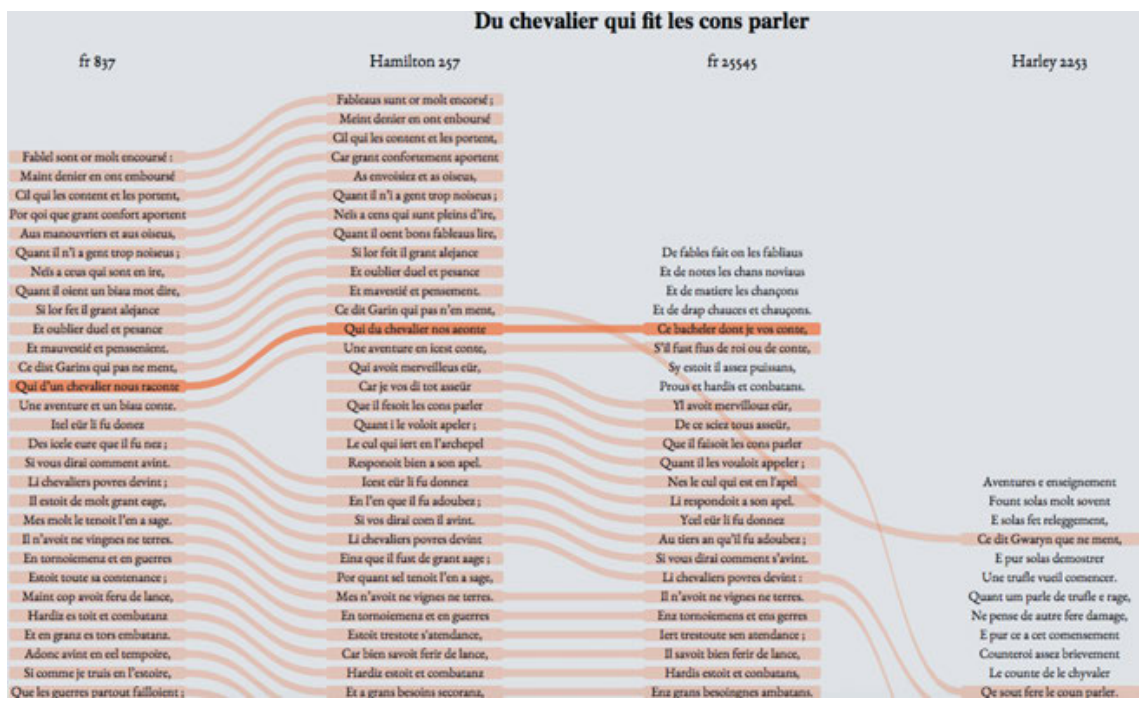

Figura 5.10: Ejemplo de Stream Graph.

Al hacer click sobre un verso, se abre una ventana con un variant graph, como se muestra en la Figura 5.11.

De esta manera, se combina un análisis de la variación a nivel estructural (las correspondencias de versos) y de frase (la variación al nivel del verso entre los diferentes testimonios). El problema de esta plataforma es que la visualización resulta muy compleja e ilegible si los textos son muy largos y existen transposiciones de versos a gran distancia (tal vez por eso los ejemplos existentes son solo de fragmentos).

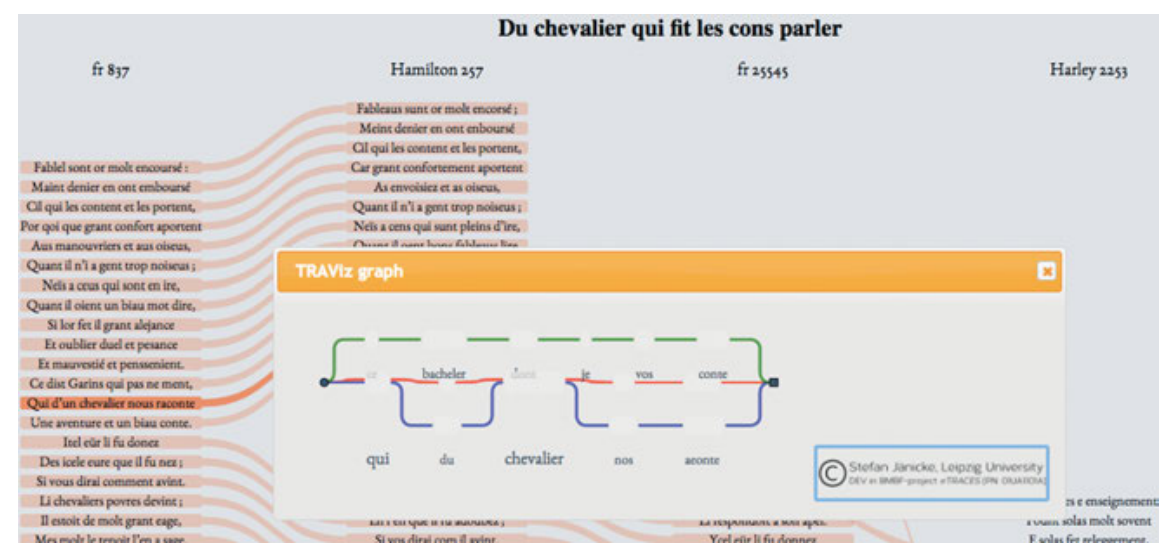

Figura 5.11: Stream Graph con grafo de variantes. 
Finalmente, una manera similar pero algo distinta de encarar el problema es la de Gustavo Fernández Riva en su edición de los relatos cortos de Konrad von Würzburg. Allí, al igual que en el stream graph, al posar el cursor sobre un verso se resaltan los versos correspondientes en los demás testimonios, pero existen varias diferencias. En primer lugar, las columnas no son estáticas, sino que pueden recorrerse ("scrollearse") de manera independiente. Al hacer click sobre un verso, todas las columnas se alinean mostrando el verso correspondiente a la misma altura, lo que permite una fácil comparación del verso y del contexto en que aparece (Figura 5.12):

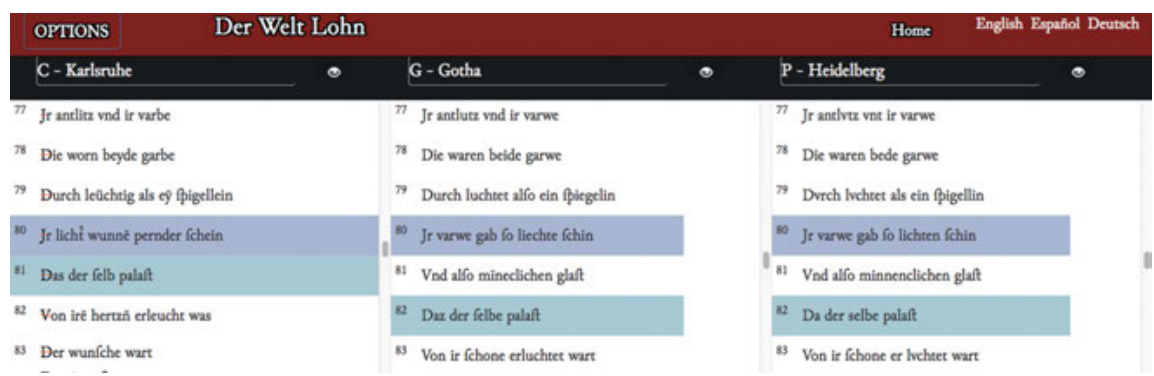

Figura 5.12: Edición de Konrad von Würzburg.

A su vez, existe la opción de representar como líneas en blanco aquellos versos que no figuren en el testimonio elegido, pero que sí existan en otras versiones (Figura 5.13).

\begin{tabular}{|c|c|c|c|c|c|c|}
\hline & OPTIONS & Loh & & & Home & hol Deutsch \\
\hline & C- Karlsruhe & $\odot$ & G - Gotha & $\odot$ & P - Heidelberg & $\odot$ \\
\hline$n$ & Jr antlitz vad ir varbe & & $n$ Jr antlute vad ir varwe & & $n$ Jr antlve vat ir varwe & \\
\hline 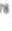 & Die worn beyde garbe & & Die waren beide garwe & & 78 Die waren bede garwe & \\
\hline 9 & Durch leikchtig als ey figellein & & Durch luchtet alfo ein fpiegelin & & 7 Dvrch lvehtet als ein figellin & \\
\hline$\infty$ & Jr licht wunnễ pernder fchein & & Jr varwe gab fo liechte fchin & & $180 \mathrm{Jr}$ varwe gab fo lichten fchin & \\
\hline s0 & & & 81 Vnd alfo mineclichen glaft & & 81 Vnd alfo minnenclichen glaft & \\
\hline 81 & Das der felb palaft & & 82 Daz der felbe palaft & & 82 Da der selbe palaft & \\
\hline
\end{tabular}

Figura 5.13: Otro ejemplo de la edición de Konrad von Würzburg.

Esta edición cuenta con un texto crítico de los relatos, en el cual se incluyen también una serie de símbolos a la derecha de ciertas líneas que indican fenómenos importantes de variación textual (versos omitidos, agregados, traspuestos o 
alterados radicalmente). ${ }^{6}$ Así, por ejemplo, un verso que se encuentra ausente en el testimonio A es acompañado por el símbolo XA (la X en color verde), mientras que un lugar donde ese testimonio tenga versos ausentes en el texto crítico estará acompañado del símbolo +A (el signo + en color rojo). El usuario puede elegir no ver estos símbolos junto al texto crítico si le resultan una molestia para la lectura, pero la ventaja de tenerlos es que permite casi inmediatamente hacerse una idea del nivel de variación presente en un fragmento determinado. Esto también permite jerarquizar la variación textual, pues solo ciertos tipos de variación se marcan con estos símbolos, mientras que para explorar las pequeñas variantes dentro de un verso es recomendable utilizar la visualización con variant graph incorporada.

La movilidad y fluidez del texto en la edición de los relatos en verso de Konrad von Würzburg intenta ofrecer una mirada que pone en primer plano la idea de mouvance (Zumthor 1972). Se transmite así la noción de que los testimonios no son estáticos, sino que son un material elástico, que presenta diferentes capas y que, por lo tanto, puede visualizarse de diferentes maneras. Se pierde, en parte, ese vistazo macroestructural de la variación, pero se gana dinamismo en la lectura (Jänicke/Wrisley 2017).

\section{Conclusiones}

Del relevamiento de las diferentes visualizaciones aquíanalizadas rescatamos algunas conclusiones generales que tenemos en cuenta para planificar nuestra edición:

1 Hay varios niveles en que se presenta variación, que requieren soluciones diferenciadas y que son difíciles de integrar. Es decir, existen variaciones a nivel del orden y la presencia de fragmentos grandes de texto (versos o párrafos), a nivel de la frase y a nivel de la palabra. Pocas plataformas logran una solución satisfactoria para esta multiplicidad de niveles. Toda visualización implica necesariamente resaltar algún aspecto en detrimento de otro. Por

6 No se ha desarrollado un criterio matemático para determinar el nivel de cambio que requiere un verso para ser considerado como radicalmente alterado. Ciertos casos son claros: versos que no comparten ninguna palabra pero que son precedidos y sucedidos por los mismos versos entran en esta categoría. Versos en los que se alteren o cambien de posición una o dos palabras claramente no entran en esta categoría. En los casos intermedios, es decir, aquellos en los cuales se comparten algunas palabras pero se cambian varias más, corresponde al juicio del editor decidir si incluirlo en estas referencias. Ya que el objetivo de estas referencias es guiar al lector, se trata de tener en cuenta qué variaciones de este tipo pueden llegar a ser interesantes para resaltar. 
ejemplo, Juxta privilegia el estudio de las variantes a nivel estructural mientras que los variant graphs lo hacen a nivel de frase.

2 La edición de textos con variantes implica una particular dialéctica entre una lectura lineal (el texto de un testimonio) y una transversal (la comparación de las variantes entre diferentes testimonios). Una buena edición sinóptica debería ofrecer la posibilidad de múltiples tipos de lectura que combinen esas dos dimensiones en diferentes proporciones.

3 El reto para el análisis de las variantes se encuentra en brindar la mayor cantidad de información posible, sin que agobie a los usuarios. Mientras el tradicional aparato crítico de las ediciones impresas solía resumir la información haciendo la reconstrucción de las variantes muy difícil, tampoco es posible para el lector humano procesar fácilmente la pluralidad que puede mostrar una edición digital. Por lo tanto, el reto está en presentar la información de una manera eficiente: compleja y abundante, pero de fácil interpretación. Se precisa para ello todavía mucho desarrollo informático.

\section{Referencias bibliográficas}

\section{Libros y artículos académicos}

Benecke, Georg Friedrich/Lachmann, Karl (1827): Iwein, der Riter mit dem Lewen. Getihtet von dem Hern Hartman Dienstman ze Ouwe. Berlín: Reimer.

Cerquiglini, Bernard (1989): Éloge de la variante: histoire critique de la philologie. Paris: Éditions du Seuil.

Discroll, Matthew James/Pierazzo, Elena (2016): Digital Scholarly Editing: Theories and Practices. Cambridge: Open Book Publishers.

Grimm, Jakob/Grimm, Wilhelm (1815): Der Arme Heinrich von Hartmann von der Aue. Aus der Straßburgischen und Vatikanischen Handschrift herausgegeben und erklärt durch die Brüder Grimm. Berlín: Realschulbuchhandlung.

Jänicke, Stefan/Wrisley, David Joseph (2017): “Visualizing Mouvance: Toward a visual analysis of variant medieval text traditions”. En: Digital Scholarship in the Humanities, 32.Suppl_2 (December), pp. 106-123. [https://doi.org/10.1093/llc/fqx033].

Lachmann, Karl (1827): Walther von der Vogelweide: Die Gedichte. Berlín: Reimer.

Nichols, Stephen (1997): “Why Material Philology? Some thoughts”. En: Zeitschrift für deutsche Philologie, 116 (Special Issue), pp 1-21.

Pierazzo, Elena (2015): Digital Scholarly Editing. Theories, models and methods. Farnham, Surrey: Ashgate.

Schmidt, Desmond/Colomb, Robert (2009): "A data structure for representing multi-version texts online". En: International Journal of Human-Computer Studies, 67.6, pp. 497-514. 
Stackmann, Karl (1964): “Mittelalterliche Texte als Aufgabe”. En: Foerste, William/Heinz Borck,

Karl (eds.): Festschrift Jost Trier. Tréveris: Böhlau, pp. 240-267.

Zumthor, Paul (1972): Essai de poétique médiévale. Paris: Seuil.

\section{Sitios Web}

CollateX: https://collatex.net/

Der Arme Heinrich digital: http://digi.ub.uni-heidelberg.de/ahd Juxta: http://juxtacommons.org/

Konrad von Würzburg Digital: https://github.com/GusRiva/konrad-von-wuerzburg MEDITE: http://obvil.lip6.fr/medite/about_medite.html

StemmaWeb: https://stemmaweb.net/stemmaweb/

Traviz: http://www.traviz.vizcovery.org/ 